\title{
Does the magnetosphere behave differently on weekends?
}

\author{
A. Karinen ${ }^{1}$, K. Mursula ${ }^{1}$, Th. Ulich ${ }^{2}$, and J. Manninen ${ }^{2}$ \\ ${ }^{1}$ Department of Physical Sciences, P.O. Box 3000, University of Oulu, FIN-90014 Oulu, Finland \\ ${ }^{2}$ Sodankylä Geophysical Observatory, Tähteläntie 62, FIN-99600 Sodankylä, Finland
}

Received: 19 November 2001 - Revised: 27 February 2002 - Accepted: 18 March 2002

\begin{abstract}
Global geomagnetic activity has been suggested to be enhanced during weekends above the weekly average after 1930. Before the 1930s, weekends and weekdays were found to be equally active. This so-called "weekend effect" was suggested to be due to power line harmonic radiation (PLHR) in the VLF range emitted by electric power lines. Since the consumption of electric power is different on weekends and weekdays, leading to different PLHR intensities, this could possibly cause the "weekend effect" in global geomagnetic activity.

In the present paper, we reanalyse the suggested "weekend effect" in global geomagnetic activity using the 69-year planetary geomagnetic $A_{p}$ index and the 131-year antipodal $a a$ index. We conclude that there is no statistically significant "weekend effect" during the interval covered by these geomagnetic activity indices. Although global geomagnetic activity is slightly enhanced on weekends from the 1930s to the 1980s, the more recent data show rather a relative decrease in global geomagnetic activity on weekends, contrary to the expected increase in the "weekend effect", due to increasing power consumption. Moreover, the weekly distribution is fairly similar in solar wind speed and global geomagnetic activity during the last 35 years, further supporting the view that the "weekend effect" is only a statistical fluctuation.
\end{abstract}

Key words. Geomagnetism and paleomagnetism (time variations, diurnal to secular) - Magnetospheric physics (planetary magnetospheres; storms and substorms)

\section{Introduction}

Electrical power lines radiate waves in the VLF (very low frequency, $300 \mathrm{~Hz}-30 \mathrm{kHz}$ ) range at harmonics of their fundamental frequency, which is usually 50 or $60 \mathrm{~Hz}$. This socalled power line harmonic radiation (PLHR) may, in principle, have effects on the behaviour of the magnetosphere.

Correspondence to: A. Karinen (arto.karinen@oulu.fi)
On the ground, the propagation of PLHR through the magnetosphere was first observed by Helliwell and Katsufrakis (1974). Subsequent studies verified that man-made VLF waves can propagate from the ground into the magnetosphere (Helliwell et al., 1975; Park, 1977; Park and Helliwell, 1978). During the past decade, low-altitude satellites have been used to study the anthropogenic PLHR in space. The AUREOL 3 satellite was the first to observe PLHR at mid-latitudes (Parrot, 1994).

Fraser-Smith (1979) reported that global geomagnetic activity had increased during weekends since the 1930s. He studied global geomagnetic activity by the 7-day superposed epoch analysis using the daily values of the $A_{p}$ and $a a$ indices in the 46.5-year (1 January 1932 - 6 June 1978) and 110-year (1 January 1868 - 31 December 1977) intervals, respectively. For the $a a$ index, separate analyses were conducted in the 63-year (1868-1930) and 42-year (1931-1972) time intervals. No increase was observed before the 1930s, but thereafter activity on weekends was enhanced. FraserSmith (1979) suggested that this so-called "weekend effect" is due to the consumption of electrical power, which is larger on weekdays than on weekends, and which has greatly increased since the 1930s. According to Fraser-Smith (1979), global geomagnetic activity is smaller on weekdays because the strong PLHR affects the magnetospheric processes so that natural disturbances are suppressed. On weekends, the activity would be higher because the smaller level of PLHR would have a smaller suppressing effect.

In this paper, we reanalyse the study of Fraser-Smith (1979) and extend it using 22.5 years of additional and more recent data on global geomagnetic activity. With an ever increasing power consumption, one could expect the "weekend effect" to be strengthened during this time. We examine the "weekend effect" in the $A_{p}$ index in Sect. 2 by the superposed epoch method and in Sect. 3, by spectral methods. In Sect. 4, we present a detailed time profile for the "weekend effect" using the 131-year $a a$ index. Section 5 presents our conclusions. 
Table 1. Mean values (m) and standard errors $\left(\sigma_{m}\right)$ of the $A_{p}$ index for each day of the week in 1932-1978, 1932-2000 and 1978-2000

\begin{tabular}{lcccccc}
\hline & \multicolumn{2}{c}{$1932-1978$} & \multicolumn{2}{c}{$1932-2000$} & \multicolumn{2}{c}{$1978-2000$} \\
& $m$ & $\sigma_{m}$ & $m$ & $\sigma_{m}$ & $m$ & $\sigma_{m}$ \\
\hline Mon & 14.66 & 0.33 & 14.96 & 0.28 & 15.77 & 0.52 \\
Tue & 14.16 & 0.31 & 14.44 & 0.26 & 15.20 & 0.45 \\
Wed & 14.28 & 0.31 & 14.49 & 0.26 & 15.12 & 0.45 \\
Thu & 14.18 & 0.33 & 14.39 & 0.26 & 14.91 & 0.41 \\
Fri & 14.21 & 0.34 & 14.51 & 0.27 & 15.16 & 0.43 \\
Sat & 14.74 & 0.35 & 14.78 & 0.28 & 14.78 & 0.46 \\
Sun & 14.73 & 0.36 & 14.70 & 0.28 & 14.69 & 0.44 \\
\hline Weekend & 14.73 & 0.25 & 14.74 & 0.20 & 14.73 & 0.32 \\
Weekday & 14.30 & 0.15 & 14.56 & 0.12 & 15.23 & 0.20 \\
\hline All days & 14.42 & 0.13 & 14.61 & 0.10 & 15.09 & 0.17 \\
\hline
\end{tabular}

\section{Superposed epoch analysis of the $A_{p}$ index}

We have calculated the weekly variation by the superposed epoch method from the daily $A_{p}$ indices for the time interval 1 January 1932 - 6 June 1978 (the interval studied by Fraser-Smith, 1979) and for two other time intervals: 1 January $1932-31$ December 2000 (the whole $A_{p}$ interval until 2000) and 1 January 1978 - 31 December 2000. Table 1 lists the average $A_{p}$ values and their standard errors for each day of the week for these three time intervals, and Fig. 1 depicts them visually. Our results for the years 1932-1978 are the same as those reported by Fraser-Smith (1979).

The average all-day $A_{p}$ value for the years $1978-2000$ (see Table 1) is larger than for the years 1932-1978, reflecting the increasing trend of global geomagnetic activity (see, e.g. Clilverd et al., 1998; Lockwood et al., 1999). However, this increase has mainly taken place on weekdays, while the weekend mean value in 1932-2000 and also in 19782000 has been roughly the same as in 1932-1978. Actually, while the interval studied by Fraser-Smith (1979) depicts roughly a $2 \sigma_{m}$ signal of enhanced weekend activity, there has been roughly a $2 \sigma_{m}$ signal of suppressed weekend activity in 1978-2000. Accordingly, global geomagnetic activity is smaller on weekends than on weekdays since the 1980s, indicating that the "weekend effect" was reversed during this time.

Consequently, the significance of the "weekend effect" during the more recent and larger $A_{p}$ interval has decreased considerably since Fraser-Smith (1979) published his studies, and is now only within a $1 \sigma_{m}$ level. This development is contrary to the idea that the "weekend effect" would be further enhanced during the last 20 years due to enhanced consumption of electric power. The small overall level of the "weekend effect" between 1932-2000 and the variation of the "weekend effect" above and below the weekly average strongly suggest that the "weekend effect" is only due to statistical fluctuation.

\section{Spectral analysis of the $A_{p}$ index}

Fraser-Smith (1979) noted pertinently that the spectral analysis of lengthy intervals of the $A_{p}$ (e.g. Fraser-Smith, 1972) and $a a$ indices (e.g. Delouis and Mayaud, 1975) do not show a well-defined 7-day spectral line. He suggested that this could be reconciled with the results of the superposed epoch method if the weekend increase only occurs intermittently in time.

We have studied the temporal occurrence of spectral power and the "weekend effect" in Fig. 2. The top panel depicts the dynamic FFT spectrum of the $A_{p}$ index for periods from 6 to 8 days. The $A_{p}$ data were split into successive annual sections, detrended and zero-padded, and the average FFT power spectral density was calculated for each annual section. The 6-8 day period part of the resulting annual spectra has been depicted along the vertical axis in a monochrome (black and white) intensity scale. The plot verifies that there are isolated enhancements of geomagnetic activity within the 6-8 day period range, but no continuous 7-day variation. Most of the variation at 6-8 days occurs during the declining phase of the solar cycle, where recurrent activity at 27-28 days dominates.

The bottom panel of Fig. 2 depicts the intensity and also the phase of the weekly variation. The daily $A_{p}$ series was first band-pass filtered within the cutoff periods of 6 and 8 days. Then the annual means for each day of the week were calculated. These values are then depicted in a monochrome (black and white) intensity scale along the vertical axis so as to demonstrate the intensity and phase of the 7-day variation within each annual section.

Figure 2 shows that in the years 1939-1942, 1946-1952 and 1957-1962, there were large variations in global geomagnetic activity at the periods from 6 to 8 days. The phase of these variations favoured weekends, as can be seen in the bottom panel. For instance, in 1953, a strong spectral line at the period of 7 days is seen in the upper panel, increasing geomagnetic activity primarily on Sundays and also on Mondays. We note that most of these do not have a period of exactly 7 days and that spectral power is fairly evenly distributed over the depicted period range. However, some of the enhancements have a period close to 7 days (see Fig. 2).

Overall, the power of geomagnetic activity variations between 6 and 8 days has become weaker after the early 1960s. The power in the 6-8 day period range was especially weak from the early 1960s to the late 1970s. During the years between 1979-1983, a few enhancements appeared primarily close to the period of 8 days. Also some weak variations at a period of about 7 days occurred during those years. In 1992-1993, strong spectral lines occurred with periods between 6 and 7 days. In 1999, there was a clear 7-day spectral line which increased geomagnetic activity during the working week.

It is interesting to note that Rodger et al. (2000) studied the so-called magnetic line radiation (MLR) for signs of anthropogenic effects. Contrary to the "weekend effect", their Fig. 4 shows a pronounced peak of MLR activity on Thurs- 


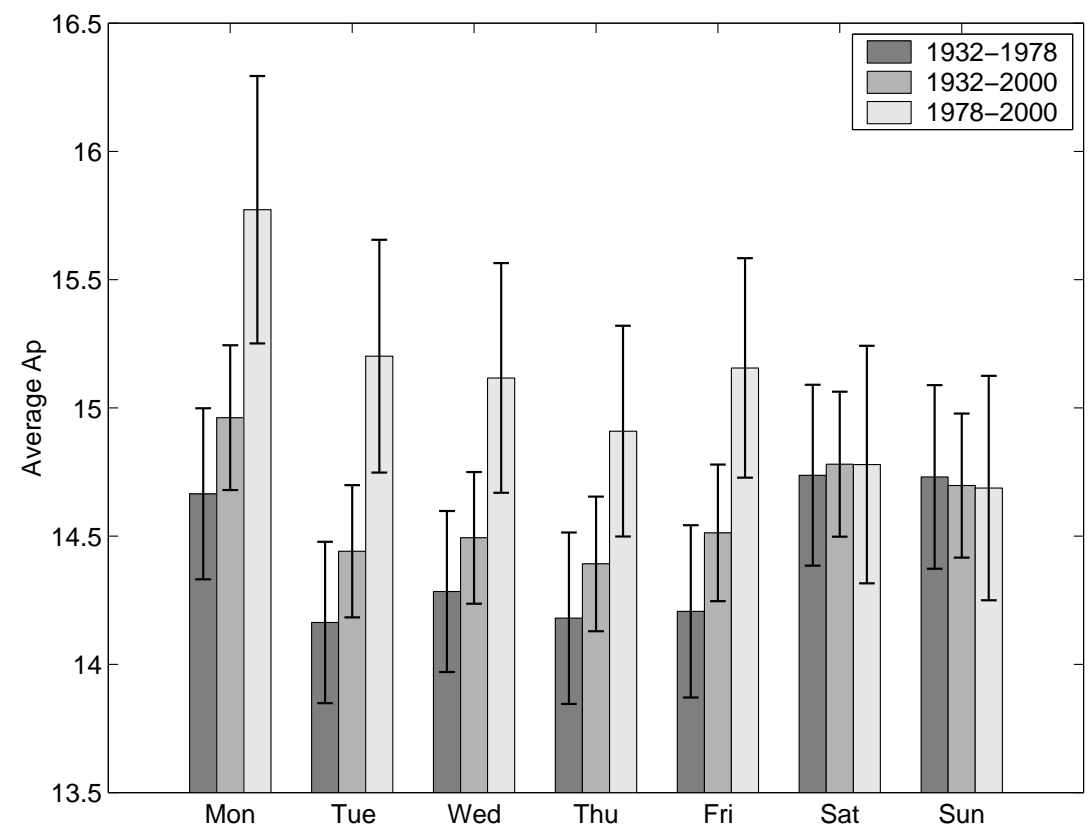

Fig. 1. Mean values and standard errors of the $A_{p}$ index for each day of the week in 1932-1978, 1932-2000 and 1978-2000.

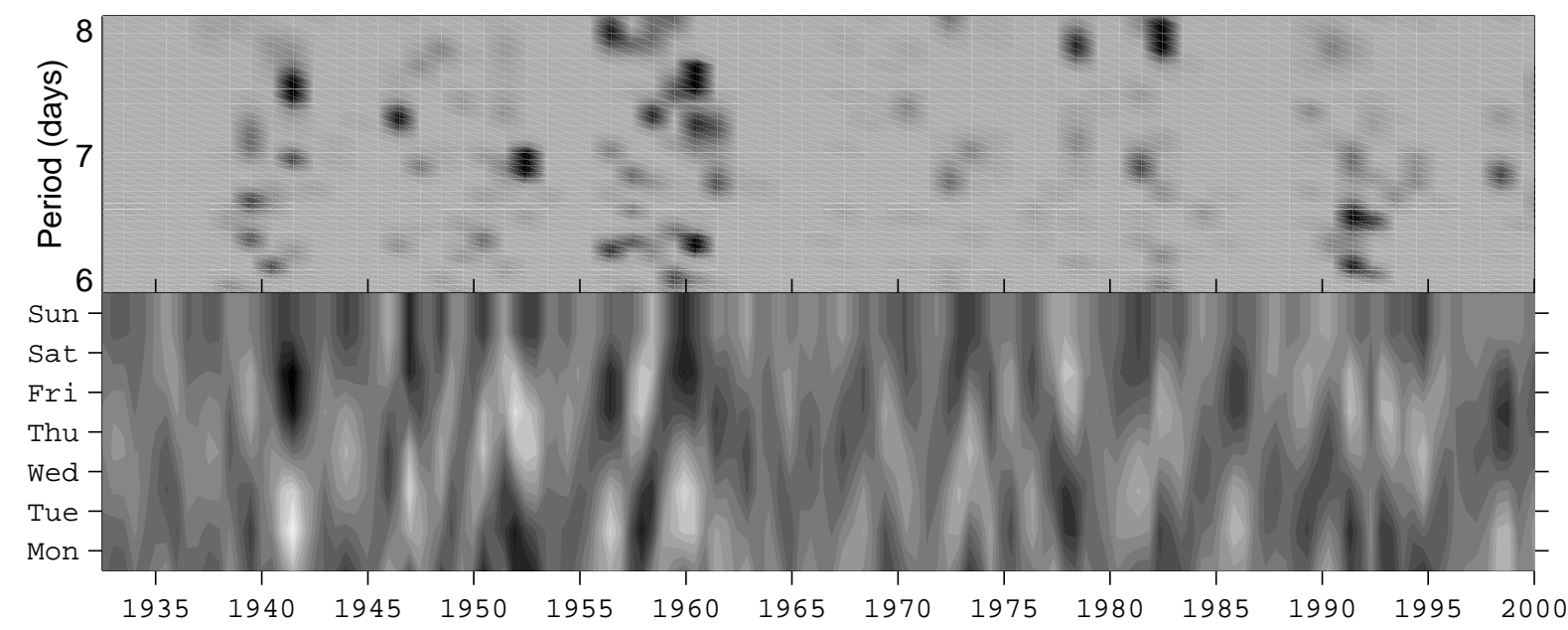

Fig. 2. (top) Dynamic FFT spectrum for the daily $A_{p}$ series in 1932-2000. Black colour represents large intensities. (bottom) Phase presentation for the weekly variation of $A_{p}$ in 1932-2000. Black (white) colour represents large, positive (negative) variations and grey colour represents the zero level.

days in the four months of VLF data of June, July, September and December 1995. We studied the same period using the $A_{p}$ index, but found neither a weekend increase nor a Thursday maximum in geomagnetic activity (see also Fig. 2).

\section{Long-term evolution of the "weekend effect"}

Long-term variations and trends can greatly affect a superposed epoch analysis. Since global geomagnetic activity is known to have increased fairly systematically during the last 100 years, this might have led to the "weekend effect" when weekly averages are compared with the values of the subsequent weekends.

In order to avoid the effect of long-term variations and trends, we have subtracted the running 13-day mean from the daily $a a$ index series to form a high-pass filtered $a a$ series. All periods longer than 13 days (e.g. solar cycle changes and 27-day variations) are thereby effectively removed from the $a a$ index.

Also, in order to avoid the artifact of the weekend being at the end of the week, we have compared the (filtered) $a a$ values for the weekend with the average level of the two adjacent working weeks. We subtracted the mean of the 10 


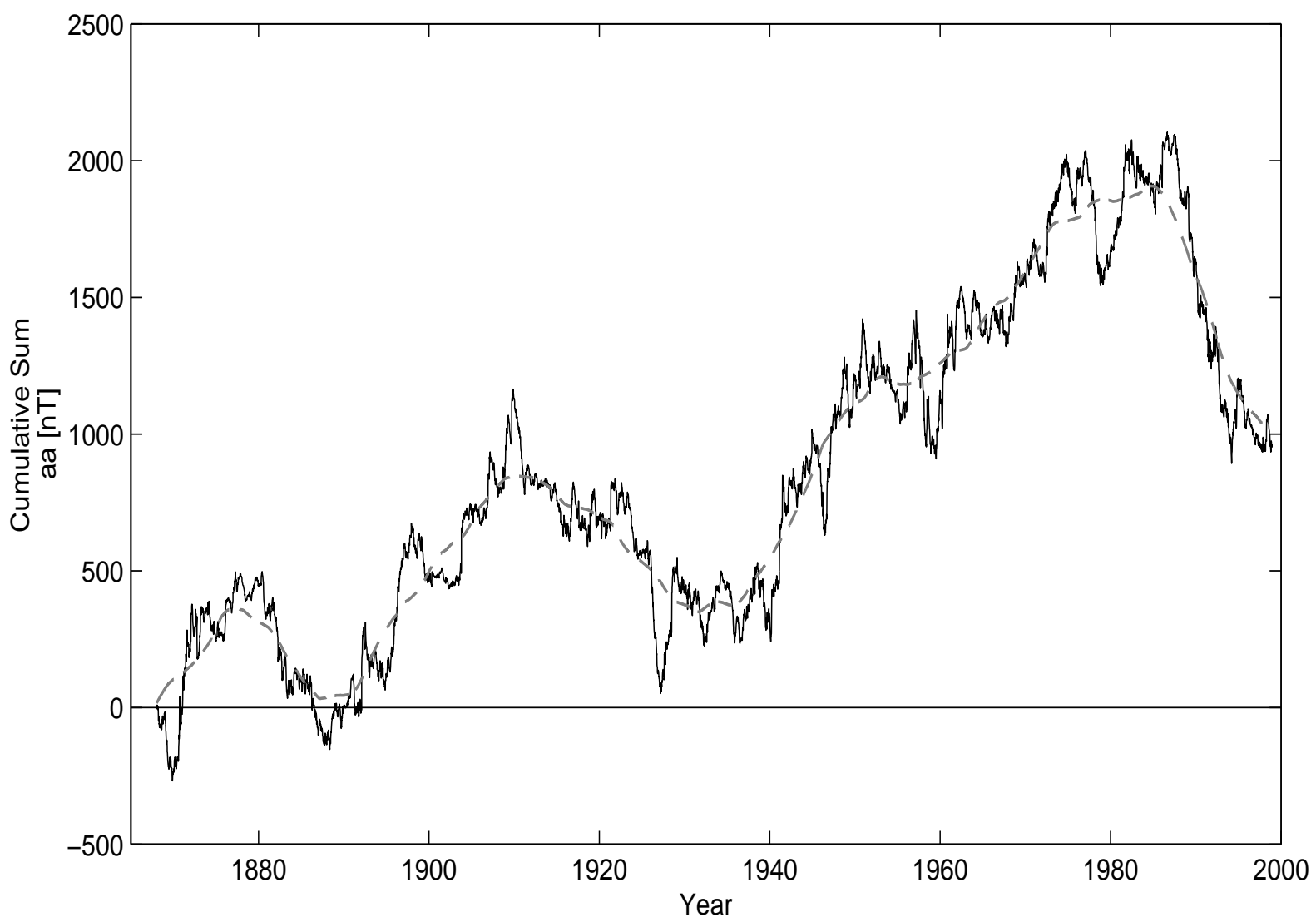

Fig. 3. Cumulative sum of weekend-weekday differences calculated from the $a a$ indices (solid curve). Running 10-year mean is depicted as a dashed curve. Horizontal line depicts the zero level of cumulative sum.

weekdays around each weekend (Saturday and Sunday) from the mean of the weekend, to form the series representing the weekend-weekday difference. In order to study the detailed time evolution of the "weekend effect", we have computed the cumulative sum of the weekend-weekday differences from the $a a$ indices for the years 1868-1998 (1 January 1868 - 31 December 1998), and plotted this curve in Fig. 3. The cumulative sum can directly depict the time evolution of any possible weekend enhancements.

Figure 3 shows that the cumulative sum has experienced three major, increasingly large and primarily positive fluctuations during the 131-year interval. The first fluctuation (1870-1890) lasted only 20 years and returned the sum to a zero level. The second, longer fluctuation (1890-1935) stopped with the cumulative sum retaining a small positive value. The third fluctuation started in the 1940s and is obviously not yet finished.

The first two fluctuations in the cumulative sum coincide with the time interval 1868-1930, which Fraser-Smith (1979) reported only a little difference between the average weekday and weekend values. The major increase in the cumulative sum from about 1940 to about 1980 corresponds to enhanced geomagnetic activity on weekends, as found by Fraser-Smith (1979) for his second time interval 1931-1972 using the superposed epoch method. Accordingly, this in- crease forms the basis of his evidence in favour of the "weekend effect". However, as seen in Fig. 3, the subsequent evolution of the cumulative sum is completely different, decreasing from the 1980s until today. This implies that global geomagnetic activity has been even weaker on weekends than on weekdays since the 1980 s.

The small size of the fluctuations is below statistical significance, as concluded earlier. Moreover, the fluctuating nature of the weekend-weekday difference, in particular the decreasing sections in 1910-1940 and from 1985 until now, is against a persistent "weekend effect" and the idea that the effect would be due to PLHR. Note that the decrease since 1985 also excludes the possibility that the "weekend effect" due to PLHR was active only in 1940-1985. Such a scenario could be envisioned if the weekly variation of power consumption was reduced recently, for example, due to increased automation (supporting a 7-day work week). Automation leads to indistinguishable weekend and weekday activity, but not to less active weekends.

Some detailed features that were found in the dynamic spectrum of the $A_{p}$ index (see Fig. 2) are also seen in the cumulative sum for $a a$. For instance, within the years 19391942, 1960-1962 and 1979-1982, the cumulative curve has rapidly increasing sections, corresponding to simultaneous spectral enhancements. 


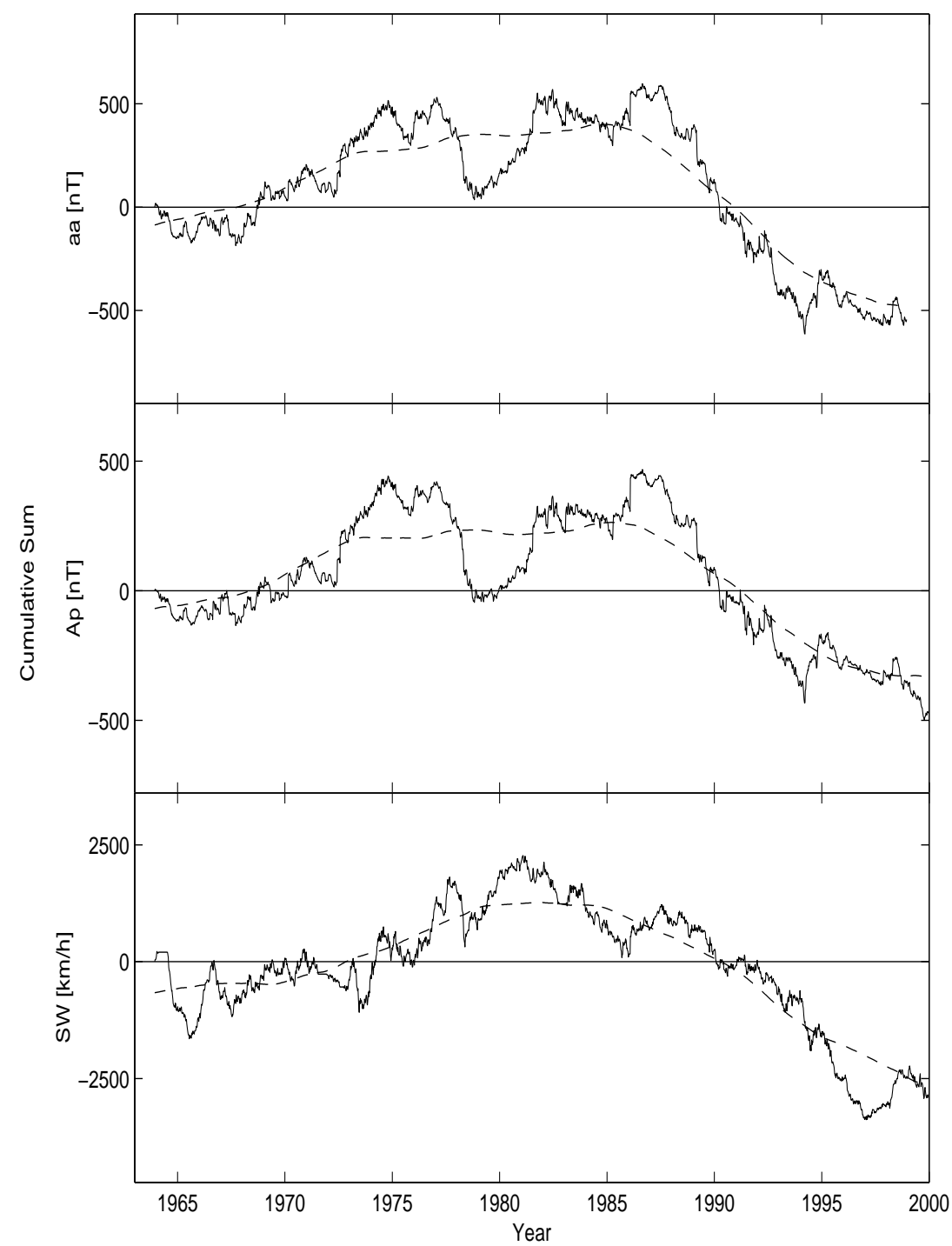

Fig. 4. Cumulative sum of weekendweekday differences for the $a a$ (top) and $A_{p}$ (center) geomagnetic indices and for the solar wind speed (bottom). Running 10-year means are depicted as dashed curves. Horizontal lines depict the zero levels of cumulative sums.

We have also studied the weekend-weekday difference in solar wind speed. Figure 4 depicts the cumulative sums of the weekend-weekday differences for the $a a$ and $A_{p}$ indices, as well as for the daily values of solar wind speed. Figure 4 shows that the weekend enhancement curves for solar wind speed and geomagnetic activity are fairly similar over the 35year interval covered. All three parameters depict a weak rise from 1965 to a maximum in about $1980-1985$ and a rapid decrease thereafter. This similarity is in accordance with the fact that global geomagnetic activity on long time scales is driven by solar wind speed.

\section{Conclusions}

In this paper, we have reanalysed the suggested evidence for a higher level of global geomagnetic activity on weekends since the 1930s (Fraser-Smith, 1979). The reason for this so-called "weekend effect" was suggested to be the different amount of power line harmonic radiation between weekends and weekdays.
We have calculated the temporal evolution of the weekendweekday difference during the last 131 years and find no systematic or statistically significant enhancement of geomagnetic activity on weekends, neither during the overall 131year interval studied, nor during the more recent decades. Instead of a systematically increasing trend, the weekendweekday difference depicts fluctuations which are, however, below statistical significance. The earlier evidence in favour of the "weekend effect" was based on the rising part of the largest to date statistical fluctuation of the weekend-weekday difference. During the last 15-20 years, the activity has become weaker on weekends than weekdays, contrary to the behaviour expected for the "weekend effect", due to power line harmonic radiation. Moreover, the weekend-weekday difference during the last 35 years is fairly similar in geomagnetic activity and solar wind speed. This is in accordance with the long-term driving of geomagnetic activity by solar wind speed and the purely statistical nature of the weekendweekday difference. 
Acknowledgement. We thank J. Kultima of Sodankylä Geophysical Observatory, Finland, for useful discussions on geomagnetic activity indices. The financial support by Finnish Graduate School in Space Physics of the Academy of Finland is gratefully acknowledged.

Topical Editor G. Chanteur thanks A. C. Fraser-Smith and M. A. Clilverd for their help in evaluating this paper.

\section{References}

Clilverd, M. A., Clark, T. D. G., Clarke, E., and Rishbeth, H.: Increased magnetic storm activity from 1868 to 1995, J. Atm. Sol. Terr. Phys., 60, 1047-1056, 1998.

Delouis H. and Mayaud, P.-N.: Spectral analysis of the geomagnetic activity index aa over a 103-year interval, J. Geophys. Res., 80, 4681-4688, 1975.

Fraser-Smith. A. C.: Spectrum of the geomagnetic activity index Ap, J. Geophys. Res., 77, 4209-4219, 1972.

Fraser-Smith, A. C.: A weekend increase in the geomagnetic activity, J. Geophys. Res., 84, 2089-2096, 1979.
Helliwell, R. A. and Katsufrakis, J.-P.: VLF wave injection into the magnetosphere from Siple Station, Antarctica, J. Geophys. Res., 79, 2511-2518, 1974.

Helliwell, R. A., Katsufrakis, J.-P., Bell, T. A., and Raghuram, R.: VLF line radiation in the Earth's magnetosphere and its association with power system radiation, J. Geophys. Res., 80, 42494258, 1975.

Lockwood M., Stamper, R., and Wild, M. N.: A doubling of the Sun's coronal magnetic field during the past 100 years, Nature, 399, 437-439, 1999.

Park, C. G.: VLF wave activity during a magnetic storm: a case study of the role of power line radiation, J. Geophys. Res., 82, 3251-3260, 1977.

Park, C. G. and Helliwell, R. A.: Magnetospheric effects of power line radiation, Science, 200, 727-730, 1978.

Parrot, M.: Observations of power line harmonic radiation by the low-altitude AUREOL 3 satellite, J. Geophys. Res., 99, 39613969, 1994.

Rodger, C. J., Clilverd, M. A., Yearby, K. H., and Smith, A. J.: Is magnetospheric line radiation man-made?, J. Geophys. Res., 105, 15981-15990, 2000. 